\title{
Analysis of the Effects of Electronic Medical Records and a Payment Scheme on the Length of Hospital Stay
}

\author{
Melaku Haile Likka ${ }^{1}$, Yukio Kurihara ${ }^{2}$ \\ ${ }^{1}$ Information Healthcare Science Course, Graduate School of Integrated Arts and Sciences, Kochi University, Kochi, Japan \\ ${ }^{2}$ Healthcare Informatics Division, Basic Nursing Department, Medical School, Kochi University, Kochi, Japan
}

Objectives: This study analyzed the effects of computerization of medical information systems and a hospital payment scheme on medical care outcomes. Specifically, we examined the effects of Electronic Medical Records (EMRs) and a diagnosis procedure combination/per-diem payment scheme (DPC/PDPS) on the average length of hospital stay (ALOS). Methods: Post-intervention changes in the monthly ALOS were measured using an interrupted time-series analysis. Results: The level changes observed in the monthly ALOS immediately post-DPC/PDPS were -1.942 (95\% confidence interval [CI], -2.856 to -1.028 ), -1.885 ( $95 \%$ CI, -3.176 to -0.593$),-1.581$ (95\% CI, -3.081 to -0.082 ) and -2.461 (95\% CI, -3.817 to 1.105 ) days in all ages, $<50,50-64$, and $\geq 65$ years, respectively. During the post-DPC/PDPS period, trends of 0.107 (95\% CI, 0.069 to 0.144 ), 0.048 ( $95 \%$ CI, -0.006 to 0.101 ), 0.183 (95\% CI, 0.122 to 0.245 ) and 0.110 (95\% CI, 0.054 to 0.167 ) days/month, respectively, were observed. During the post-EMR period, trends of -0.053 ( $95 \% \mathrm{CI},-0.080$ to -0.027 ), -0.093 (95\% CI, -0.135 to -0.052 ), and -0.049 (95\% CI, -0.087 to -0.012 ) days/month were seen for all ages, 50-64 and $\geq 65$ years, respectively. Conclusions: The increasing post-DPC/PDPS trends offset the decline in ALOS observed immediately post-DPC/PDPS, and the observed ALOS was longer than the counterfactual at the end of the DPC/PDPS study periods. Conversely, due to the downward trend seen after EMR introduction, the actual ALOS at the end of the EMR study period was shorter than the counterfactual, suggesting that EMRs might be more effective than the DPC/PDPS in sustainably reducing the LOS.

Keywords: Electronic Medical Records, Longitudinal Studies, Length of Stay, Quality of Health Care, Prospective Payment System

Submitted: January 28, 2021

Revised: August 26, 2021

Accepted: October 4, 2021

\section{Corresponding Author}

Melaku Haile Likka

Information Healthcare Science Course, Graduate School of Integrated Arts and Sciences, Kochi University, 185-1 Kohasu, Oko-Cho, Nankoku-Shi, Kochi 783-8505, Japan. Tel: +81-80-9834-2221, Email: likkamelaku@gmail.com (https://orcid.org/0000-0001-5650728X)

This is an Open Access article distributed under the terms of the Creative Commons Attribution Non-Commercial License (http://creativecommons.org/licenses/bync/4.0/) which permits unrestricted non-commercial use, distribution, and reproduction in any medium, provided the original work is properly cited.

(C) 2022 The Korean Society of Medical Informatics

\section{Introduction}

Health systems have been taking measures to tackle escalating healthcare costs and ensure the delivery of high-quality, including the introduction of new hospital reimbursement schemes, such as the diagnostic related group-based prospective payment system (DRG/PPS), and the implementation of Electronic Medical Record (EMR) systems. The DRG/PPS is an activity-based hospital funding system in which clinically similar groups of patients are classified by their treatments and costs, and care providers are reimbursed a flat amount per diagnosis group. Activity-based funding (ABF) schemes, such as the DRG/PPS, have been introduced to increase 
the efficiency of healthcare services [1]. The reduction of the length of stay (LOS) of inpatients is a strategy used to achieve healthcare outcomes efficiently [2].

In 2003, Japan introduced a variant of DRG/PPS called the diagnostic procedure combination/per-diem payment system (DPC/PDPS), in which per-diem based payments for medical services are pre-determined according to the classification of each inpatient activity by diagnosis, procedure, and severity. Medical charges under DPC/PDPS consist of inclusive (DPC) and fee-for-service (FFS) components. The DPC component is used to pay for the basic hospital stays and treatments with $\leq 1,000$ points and is calculated by multiplying the per-diem payment rate set for each DPC group by the LOS and a coefficient specific to each medical institution. The FFS part is used to calculate the reimbursements for medical care and procedures with $>1,000$ points. If the admission takes longer than the predetermined duration, the DPC scheme is no longer used, and the costs are covered through FFS. The per-diem allocated to admissions with shorter LOS is higher than that of admissions with longer LOS and, consequently, hospitals are expected to shorten the average LOS (ALOS) [3]. The combination of both DRG and FFS schemes, per diem-based payment, and the adjustment of the DPC groups for LOS in calculating the medical costs makes the DPC/PDPS different from the DRG/PPS and unique to Japan [4] and the Republic of Korea (ROK) [5].

Although most of the literature on the effectiveness of DRG-based schemes in Japan and elsewhere showed a positive contribution of the schemes to reducing healthcare costs and LOS [5-11], a few of them revealed they did not bring significant changes $[12,13]$.

Meanwhile, to enhance the quality of healthcare, improve efficiency and patient safety, hospitals have been adopting health information technologies (HITs) such as computerized physician order entry (CPOE) and EMRs in recent decades [14]. An EMR is defined as "an electronic version of personal medical data maintained by the healthcare provider, and incorporates all the key administrative and clinical data relevant to that person's care under a specific provider" [15]. The Japan Association of Medical Informatics (JAMI) categorizes EMR implementation in Japan into two levels: basic EMRs, which support order transmission; and paperless EMRs, which support order transmission, allow reference to the order results in all application areas, and enable electronic management of all types of information constituting the medical record [16].

Theoretically, EMRs are supposed to improve the quality of care and reduce healthcare costs and LOS by enabling prompt information sharing and retrieval, reducing medication errors, and curbing unnecessary tests and procedures. However, studies focusing on the effects of EMRs have reported inconsistent results [17-24]. In Japan, EMR-related studies have focused on levels of adoption and factors affecting adoption [25-27].

The existing evidence on the relationship between LOS or cost of healthcare and DPC/PDPS in Japan has been produced based on temporary or short-term effects that were observed shortly after implementation of the scheme. No studies in Japan have considered changes in postintervention trends that could demonstrate the long-term effects of the scheme. Furthermore, the effects of EMRs on LOS have yet to be explored in Japan. The existing literature also does not provide evidence on the comparative effects of both the payment system and electronic interventions on hospital LOS or cost. To address these gaps and supplement the existing research on the relationships between EMR or $\mathrm{ABF}$ schemes and LOS, we measured and compared the short- and long-term effects of the adoption of EMRs and the DPC/PDPS on monthly ALOS in an advanced treatment hospital. In this study, short-term effects refer to the changes observed in the level of monthly ALOS (in days) immediately after implementation; and the long-term effects are the changes observed in the trends of the monthly ALOS (in days/month) during the post-implementation period.

The purpose of this study was to analyze the effects of the computerization of medical information systems and a hospital payment scheme on an outcome related to medical care. We hypothesized that, as the main purpose of DPC/ PDPS is to reduce the LOS, it would be more effective than EMRs in reducing the ALOS in the short and long term. The research questions of this study were: (1) Do EMRs and the reimbursement scheme contribute to reducing the monthly ALOS immediately post-implementation? (2) Do both interventions alter the trend of monthly ALOS post-implementation? (3) Which of the interventions is more effective in reducing the monthly ALOS immediately and sustainably?

\section{Methods}

\section{Study Setting and Data}

The study was conducted in the inpatient departments of an advanced treatment university hospital in Japan. The hospital was established in the early 1980s and has been equipped with a CPOE since its establishment. The hospital subsequently incorporated computerized medical imaging and nursing record systems into the CPOE and in 2007, it introduced a pa- 
perless EMR system that curbed paper-based activities. The hospital also became part of the DPC/PDPS in June 2003.

Deidentified data that consisted of sex, age at admission, LOS, and admission year and month of inpatients admitted in 2000-2010 were obtained from the hospital's information center. Cases with LOS $>90$ days (a small percentage of inpatients who were primarily admitted for geriatric, psychiatric, hematologic, and gastrointestinal conditions) were excluded as such cases could not be reasonably affected by the interventions studied. Finally, inpatients were grouped by admission year and month and their LOS was averaged to extract the monthly ALOS.

The differences in the monthly ALOS before and after the introduction of the DPC/PDPS and EMR system were examined to determine their effects on changes in levels and the post-intervention trends by controlling for the existing trends caused by factors other than the interventions investigated. The monthly ALOS data from January 2000 to December 2006 (84 months) and January 2003 to December 2010 (96 months) were used to evaluate the impact of the DPC/PDPS and EMR, respectively. The 41 months of December 2000 to May 2003 and the subsequent 43 months were taken as the pre-DPC/PDPS and post-DPC/PDPS periods, respectively. Similarly, January 2003 to December 2006 and January 2007 to December 2010 were used as pre-EMR and post-EMR periods, respectively. The overlapping of the pre-EMR periods with the post-DPC/PDPS does not affect the estimates of the level changes and post-EMR trends as the pre-EMR events are controlled by the coefficient that represents the existing (pre-intervention) trends $\left(\beta_{1}\right.$, explained in Section II-3). As the monthly ALOS was significantly different among inpatients in various age categories, the statistical analyses were performed on four sets of data (all ages and subgroups of $<50,50-64$, and $\geq 65$ years of age at admission).

\section{Study Design}

An interrupted time-series (ITS) analysis, a regression-based quasi-experimental study design $[28,29]$ was used to evaluate the effectiveness of the interventions. Three independent variables were created to perform the analysis:

T: months covering the study periods sequentially, increasing by 1 each month,

$\mathrm{X}_{\mathrm{t}}$ : a dummy variable indicating the pre-intervention period (coded 0$)$ or the post-intervention period ( coded 1) for each intervention, and

$\mathrm{T}_{\mathrm{tr}}$ : the post-intervention period, which was assigned a value of 0 for all pre-intervention series and increased with a unit representing months after the intervention.

\section{Statistical Analysis}

The following segmented regression model was used to obtain the coefficients:

$$
\text { Monthly ALOS }=\beta_{0}+\beta_{1} T+\beta_{2} X_{t}+\beta_{3} T_{t r}+\varepsilon_{t}
$$

where $\beta_{0}$ represents the baseline monthly ALOS at $\mathrm{T}=0, \beta_{1}$ is the change in outcome associated with $\mathrm{T}$ representing the underlying pre-intervention (existing) trends, $\beta_{2}$ is the level change following the intervention, and $\beta_{3}$ indicates the trend change following the intervention.

Plots of the autocorrelation function (ACF) and partial autocorrelation function (PACF) of the residuals of the ordinary least square regression models of each set of data were used to diagnose autocorrelation, and the relevant statistical tests were used to diagnose heteroscedasticity and seasonality of the series. The statistical and visual diagnostics showed the presence of serial correlation and seasonality in the series. Hence, the serial correlation was adjusted by employing generalized least square (GLS) regression. The GLS models with first-order serial correlation (autoregression moving average $(\operatorname{ARMA}(1,0))$ were selected for all sets of data among the several GLS models constructed based on the lags indicated by the ACF and PACF plots. The Bayesian information criterion was used to select the best model. The seasonality of the series was adjusted by introducing the Fourier series to the selected GLS model. The $95 \%$ confidence interval (CI) of the estimates was used to establish associations. Microsoft Structured Query Language Server Management Studio version 17.9.1 was used to query and manage the data, and $\mathrm{R}$ version 4.0.3 was used for analyses.

\section{Ethical Consideration}

The study protocol for this study was submitted for ethical consideration by the Ethics Review Board of the university and, as personally identifiable information was not included, the Board decided to waive ethical review.

\section{Results}

\section{Characteristics of the Participants and Changes in the ALOS during the Study Period}

Between 2000 and 2010, a total of $86,769(43,962 ; 50.666 \%$ females) admissions for all causes with LOS $\leq 365$ days took place, with an ALOS of 23.036 days. Among them, 84,026 (96.839\%) had an LOS $\leq 90$ days, from whom the monthly ALOS was derived. The ALOS of these cases between 2000 and 2010 was 19.327 days (Table 1, Figure 1).

The ALOS and its post-intervention changes were not significantly different between females and males. However, 


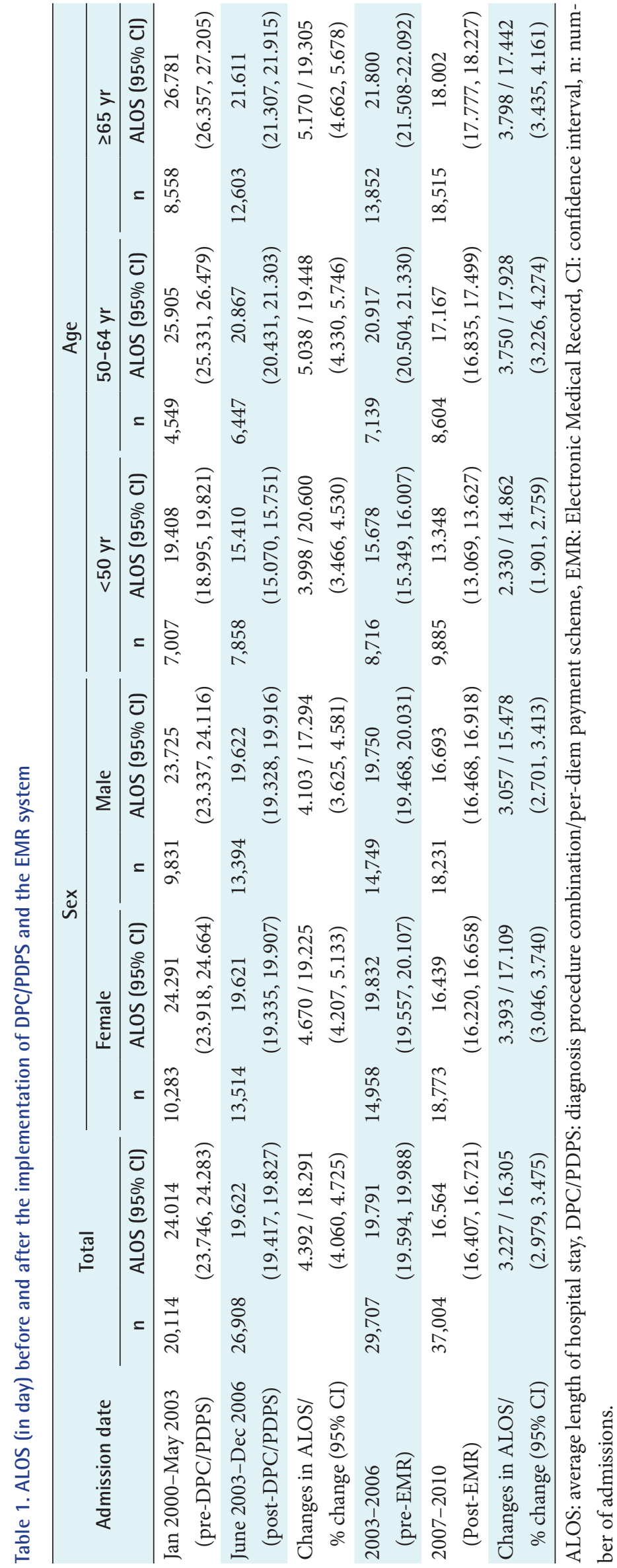




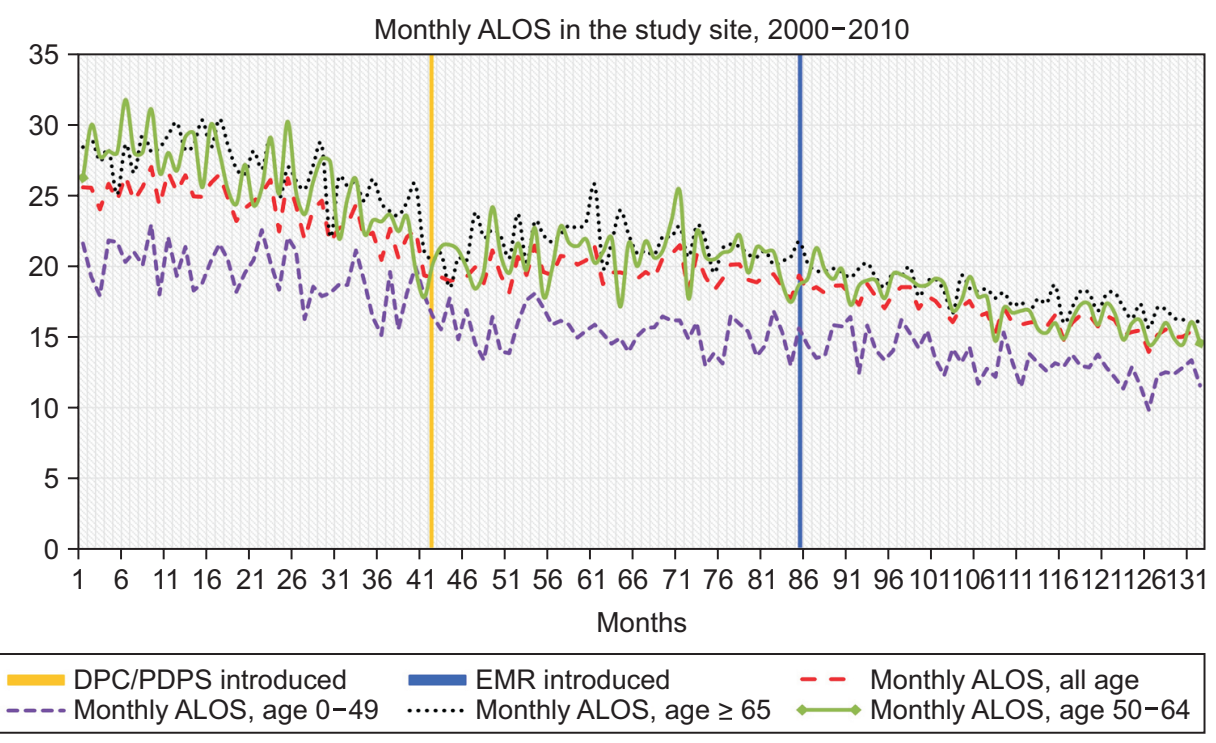

Figure 1. Monthly ALOS in the hospital during the study period, 2000-2010. ALOS: average length of hospital stay, DPC/ PDPS: diagnosis procedure combination/per-diem payment scheme, EMR: Electronic Medical Record.

inpatients $<50$ years of age had shorter LOS than older ones. The ALOS of inpatients $<50,50-64$ and $\geq 65$ years of age pre-DPC/PDPS introduction was 19.408 (95\% CI, 18.99519.821), 25.905 (95\% CI, 25.331-26.479), and 26.781 (95\% CI, 26.357-27.205) days, respectively; post-DPC/PDPS, they were 15.410 (95\% CI, 15.070-15.751), 20.867 (95\% CI, 20.431-21.303), and 21.611 (95\% CI, 21.307-21.915) days, respectively. The proportional decline in LOS following DPC/PDPS implementation was larger in the $<50$ years age group $(20.600 \%)$ than in the $\geq 65$ years age group $(19.305 \%)$ (Table 1).

The ALOS of the $<50,50-64$, and $\geq 65$ years age groups pre-EMR was 15.678 (95\% CI, 15.349-16.007), 20.917 (95\% CI, 20.504-21.330), and 21.800 (95\% CI, 21.508-22.092) days, respectively. Post-EMR, it was 13.348 (95\% CI, $13.069-$ 13.627), 17.167 (95\% CI, 16.835-17.499), and 18.002 (95\% CI, 17.777-18.227) days, respectively. The reduction in the LOS post-EMR was significantly larger in the $\geq 65$ years age group $(17.442 \%)$ than in the $<50$ years age group $(14.862 \%)$ (Table 1).

\section{Effects of DPC/PDPS on Monthly ALOS}

The segmented regression model showed that the ALOS decreased shortly post-DPC/PDPS implementation by 1.942 (95\% CI, -2.856 to -1.028$),-1.885$ (95\% CI, -3.176 to $-0.593),-1.581$ (95\% CI, -3.081 to -0.082$)$, and -2.461 (95\% CI, -3.817 to -1.105$)$ days for all ages and the $<50,50-64$ and $\geq 65$ years age groups, respectively (Table 2 ). During the 41 months of the pre-DPC/PDPS period, there were downward trends in the monthly ALOS in all groups--0.117 (95\% CI, -0.145 to -0.090$),-0.078$ (95\% CI, -0.117 to -0.039 ), -0.186 (95\% CI, -0.232 to -0.141 ), and -0.125 (95\% CI,
-0.167 to -0.084$)$ days/month-for all ages and the $<50$, $50-64$, and $\geq 65$ years age groups, respectively. However, the implementation of the scheme was associated with an upward trend in ALOS-0.107 (95\% CI, 0.069-0.144), 0.183 (95\% CI, 0.122-0.245), and 0.110 (95\% CI, 0.054-0.167) days/month-for all ages, the 50-64 years age group, and the $\geq 65$ years age group, respectively, during the 43 months post-DPC/PDPS (Table 2).

\section{Effects of EMR on Monthly ALOS}

Like the pre-DPC/PDPS period, the pre-EMR period also saw a negative trend in monthly ALOS, except for the age group of 50-64 years ( $p=0.4084$ ) (Table 3$)$. The 48 months of the post-EMR period saw significant downward trends in the monthly ALOS in all groups except the $<50$ years age group ( $p=0.5889$ ) in which the ALOS declined by rates of -0.053 (95\% CI, -0.080 to -0.027 ), -0.093 (95\% CI, -0.135 to -0.052 ), and -0.049 ( $95 \% \mathrm{CI},-0.087$ to -0.012 ) days/ month for all ages, the 50-64 years age group, and the $\geq 65$ years age group, respectively. However, no short-term reduction in ALOS was observed post-EMR in all groups (Table 3).

\section{Discussion}

In this study, it has been revealed that the DPC/PDPS was associated with a reduction of ALOS by 1.942 days $(p<0.0001)$ shortly post-implementation. This finding has been corroborated by other studies from other countries implementing ABF [5-9] and Japan [10,11]. A meta-analysis showed that the DRG-based scheme was associated with a shorter LOSpooled effect, $-8.07 \%$ (95\% CI, $-13.05 \%$ to $-3.10 \%$ ) [7]. A review of the effectiveness of $\mathrm{ABF}$ systems also revealed that 

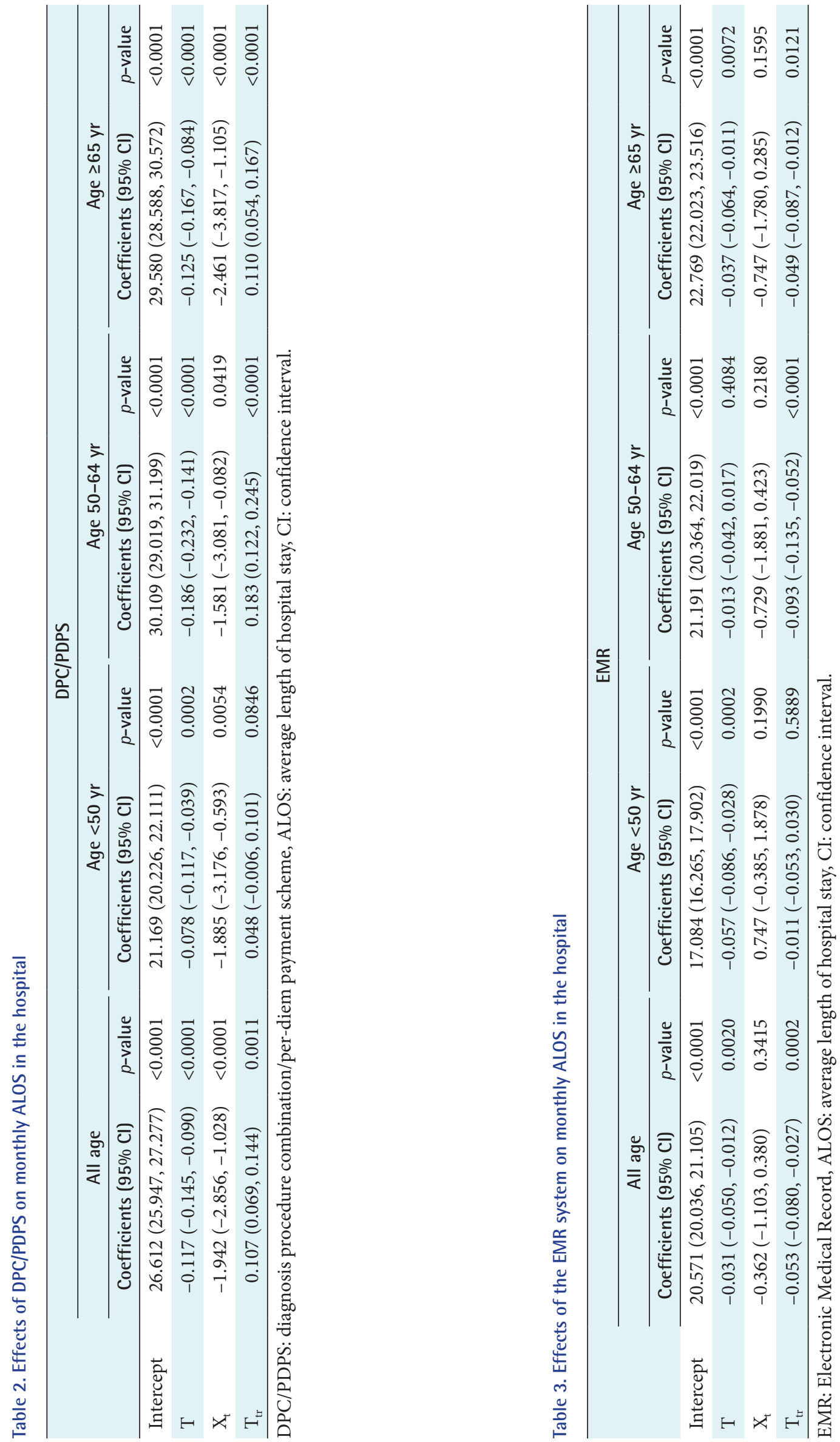
these schemes decreased the LOS in England, Germany, Ireland Finland, and public hospitals in France [6].

In the ROK, where three hospital reimbursement systems-FFS, DRG/PPS (since 2002), and Korean diagnosis procedure combination (KDPC) (since 2009) [5] - are used, the $\mathrm{ABF}$ systems have been consistently shown to reduce the LOS $[5,8,9]$. The implementation of DRGs in the field of obstetrics and gynecology at Korean tertiary hospitals led to reductions in the LOS for inpatients receiving cesarean sections, hysterectomies, adnexectomies, and simultaneous surgical procedures, in whom the LOS declined from $8.0 \pm$ $6.9,7.4 \pm 3.5,6.3 \pm 3.6,6.6 \pm 3.2$ days pre-DRG to $6.0 \pm 2.3$, $6.4 \pm 2.7,6.2 \pm 4.0$, and $5.8 \pm 2.4$ days post-DRG, respectively $(p<0.001)$ [8]. Another study also showed that the DRG payment system led to a reduction of LOS in the ROK [9]. A comparison of the effectiveness of the DRG and KDPC on the LOS showed that, although both schemes were designed to reduce the LOS, the reduction was stronger for the DRG than for the DPC payment system [5].
In Japan, an analysis of administrative data for acute myocardial infarction showed that DPC/PDPS significantly reduced the LOS by 2.29 days ( $95 \% \mathrm{CI},-3.71$ to -0.88 ) [10]. Similarly, among hip-fracture inpatients at the Japanese Red Cross medical centers, the reduction of ALOS among DPC/ PDPS-implementing hospitals (30.1 days pre-DPC/PDPS to 23.5 days post-DPC/PDPS) were significantly higher than those hospitals without DPC/PDPS (38.5 to 36.4 days during the same periods) ( $p=0.003)$ [11]. As the primary purpose of ABF schemes is to reduce LOS and costs, the reported results are in line with the expected outcomes. However, a few studies have reported results inconsistent with the current study, finding that the DRG-based schemes were not associated with the LOS $[12,13]$.

In this study, there were existing downward trends in the monthly ALOS of $-0.117(p<0.0001),-0.078(p=0.00012)$, $-0.186(p<0.0001)$, and $-0.125(p<0.0001)$ days/month for all ages and the $<50,50-64$ and $\geq 65$ years age groups during the pre-DPC/PDPS period, respectively (Table 2). These pre-

\section{Observed and counterfactual monthly ALOS before and after DPC/PDPS implementation}
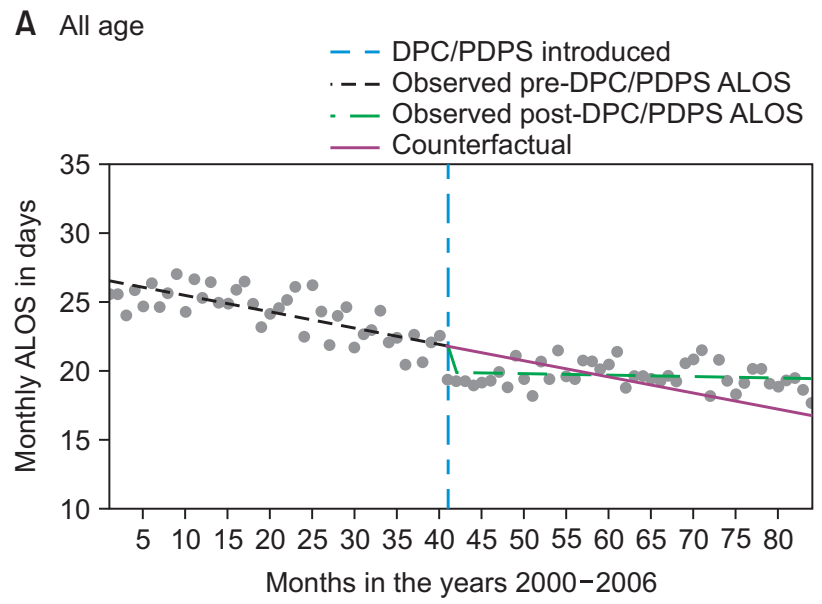

C Age 50-64 years

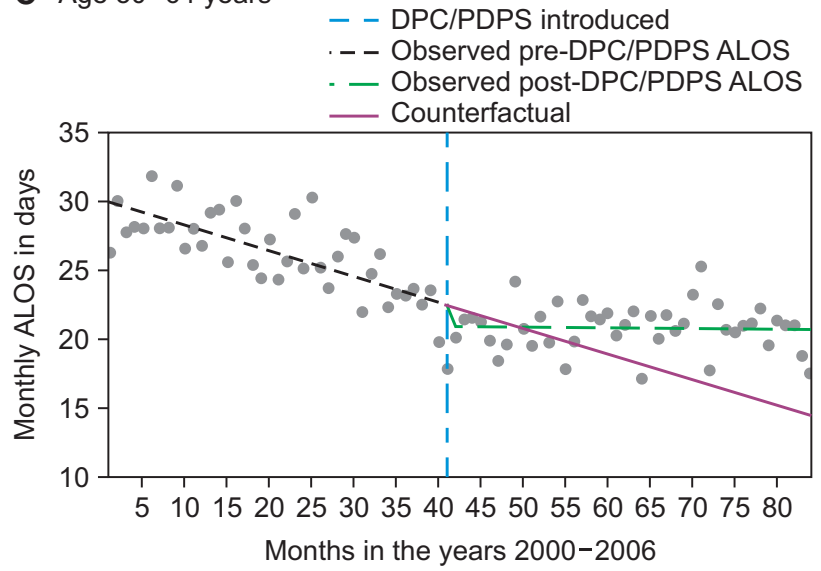

B Age $<50$ years
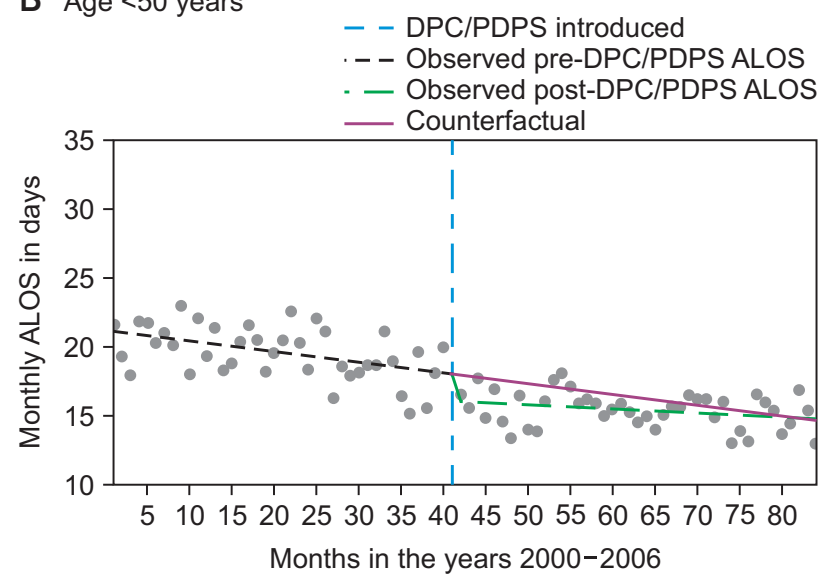

D Age $\geq 65$ years

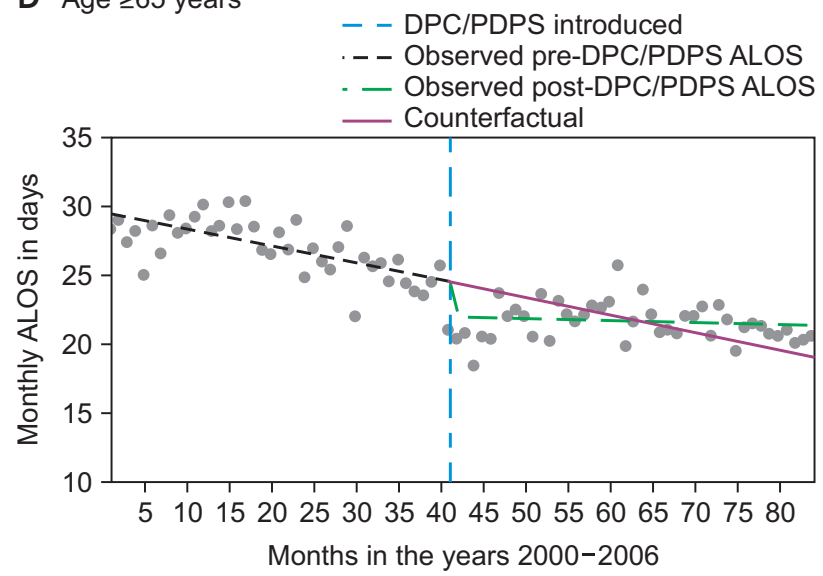

Figure 2. Observed pre- and post-DPC/PDPS monthly ALOS and the counterfactuals: (A) all age, (B) age <50 years, (C) age 50-64 years, (D) age $\geq 65$ years. DPC/PDPS: diagnosis procedure combination/per-diem payment scheme, ALOS: average length of hospital stay. 
DPC/PDPS trends are attributed to factors other than the intervention studied. Attempts were made to reduce the LOS in Japan even before the introduction of the DPC/PDPS [3] and the observed existing trend could be related to these efforts.

However, the pre-DPC/PDPS downward trends were diminished by the upward trend in the ALOS observed during the post-DPC/PDPS period. The DPC/PDPS initiated an upward trend of monthly ALOS by 0.107 days/month (all ages) post-implementation $(p<0.0001)$. The rate of increment in the trend of ALOS was higher in the age group of 50-64 years $(0.183$ days/month, $p<0.0001)$ (Table 2$)$. Hence, the observed ALOS a few months after the implementation of this intervention was longer than the counterfactual in all groups (Figure 2). This finding suggests that the DPC/PDPS might have had a counterproductive effect on efforts to reduce the LOS in the long term.

Regarding the effect of EMRs on ALOS, unlike in the case of DPC/PDPS, no significant changes in the monthly ALOS were observed in any of the age groups immediately post-
EMR introduction (Table 3), but a negative existing trend was observed during the pre-EMR period, except for the age group of $50-64(p=0.4084)$ (Table 3$)$. The post-EMR period also saw statistically significant negative trends in the monthly ALOS in inpatients of age $\geq 50$. After EMR adoption, the monthly ALOS significantly decreased in the groups of all ages, $50-64$ years, and $\geq 65$ years by rates of -0.053 $(p=0.0002),-0.093(p<0.0001)$, and $-0.049(p=0.0121)$ days/month, respectively. Consequently, the observed ALOS during the study periods was significantly shorter than the counterfactual for inpatients age $\geq 50$ (Figure 3 ). However, no significant association was observed between EMR adoption and the trend or level of ALOS for inpatients $<50$ years (Table 3). This could have been because the LOS was already short in that age group and no further reduction could be achieved.

Similar studies employing the same study design as the current one has reported similar findings, in which EMRs did not change the ALOS at hospitals instantly post-EMR,

\section{Observed and counterfactual monthly ALOS before and after EMR implementation}

A All age

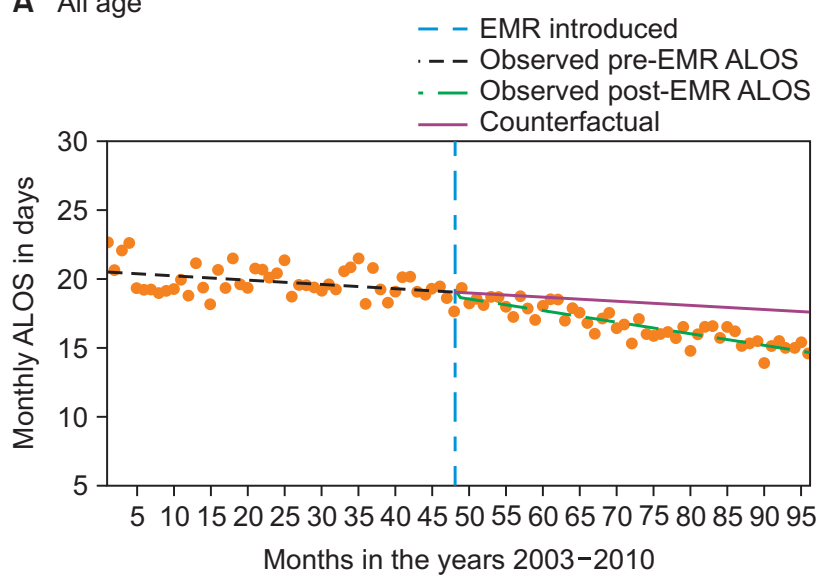

C Age 50-64 years

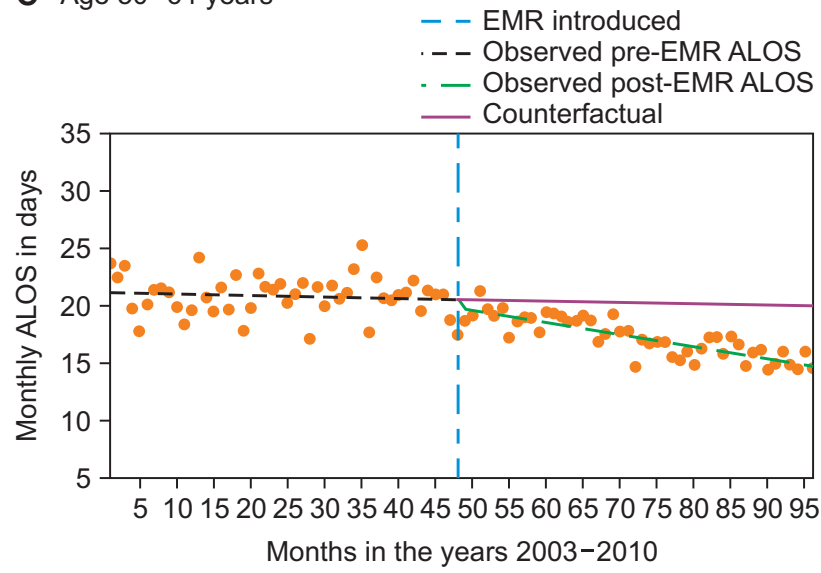

B Age $<50$ years

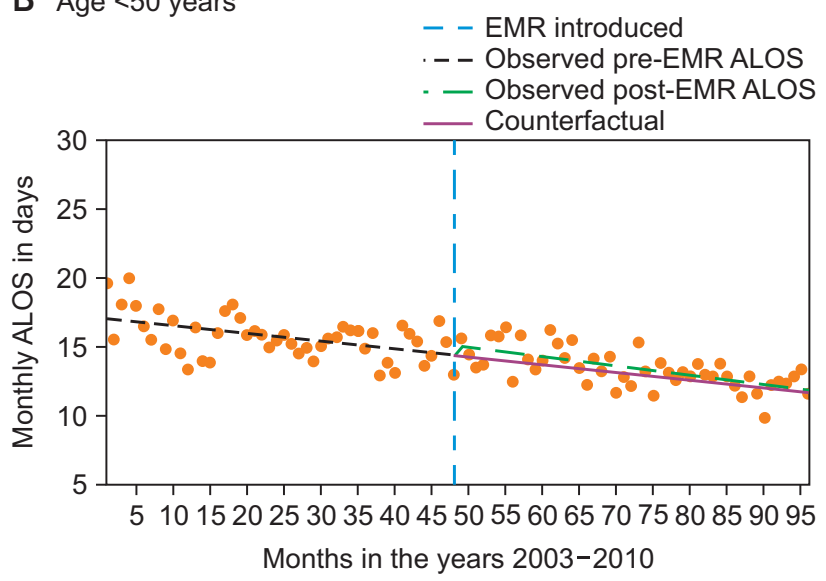

D Age $\geq 65$ years

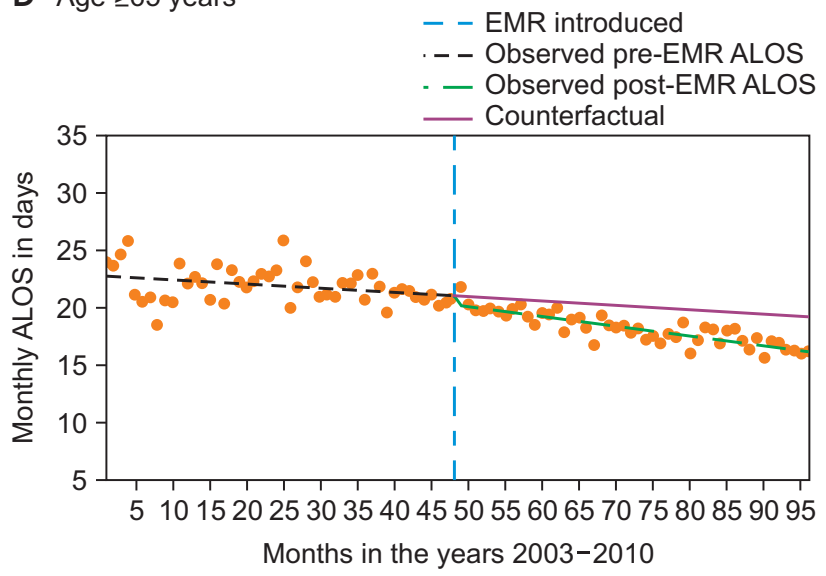

Figure 3. Observed pre- and post-EMR monthly ALOS and the counterfactuals: (A) all age, (B) age <50 years, (C) age 50-64 years, (D) age $\geq 65$ years. EMR: Electronic Medical Record, ALOS: average length of hospital stay. 
but led to a reduction in the long-term trends of the ALOS $[22,23]$. Two studies from two large hospitals in China reported that the trend changes in ALOS reversed from 0.004 days/month to -0.070 days/month; $p<0.001$ ) [22] and from 0.027 to -0.043 bed-days/month in the post-EMR period $(p<0.001)$ [23]. In acute care facilities in California, the overall LOS was reduced by $3 \%$ for all inpatients admitted at hospitals that used EMRs meaningfully compared to those without meaningful use of EMRs [21].

Nevertheless, other studies documented that EMRs had no significant impacts on the LOS. A meta-analysis concluded that electronic interventions did not have significant effects on the LOS and costs of medical care [17]. Primary studies [18-20] also reported that the lack of association between EMR adoption and LOS. A comparison of the LOS between hospitals showed that the LOS was not significantly different between hospitals with HIT experience for $<5$ years and $>5$ years [18]. It was also demonstrated that the ALOS was not different at Medicare hospitals with and without EMRs [19]. A study that compared the ALOS and other patient outcomes of medical and surgical inpatients in six states in the United States implementing no, partial, and full EMRs found that there were no significant differences in patient outcomes among the hospitals [20].

A reason for the negative trend during the post-EMR period but no change in the level of ALOS shortly postimplementation could be that the introduction of new technologies requires substantial time to get used to and to be integrated into the institutional culture before realizing the anticipated outcomes. Furthermore, the primary purpose of EMRs is to enhance the quality of care and patient safety by providing complete access to patient information to the professionals involved in care, reducing medical errors, improving care coordination, reducing workloads, and improving decision-making processes. Reductions in LOS and the cost of care are realized after these quality outcomes are achieved. As a result, the EMR-induced reduction in LOS may not be achieved instantly following its adoption.

Through a comparison of the observed changes in the LOS during the DPC/PDPS and EMR evaluation periods, we have shown that the short-term effect of the reimbursement system in reducing ALOS was offset by the post-DPC/PDPS upward trends associated with the scheme. In contrast, EMR implementation was associated with a reduction of LOS over the long term, hinting that EMRs might be more effective in sustainably reducing the LOS.

Nonetheless, as EMRs affect the activities involved in medical care, the use of DPC/PDPS could be boosted follow- ing the adoption of an EMR. Although there is no evidence showing the interaction of HITs and the payment schemes to improve medical care outcomes, the observed change in the trends of ALOS in this study could result from improvements in the DPC/PDPS practice because of EMR adoption. Further studies are needed to demonstrate if there is such an interaction between the two systems and to establish a causal relationship between EMR implementation and LOS.

This study showed that introduction of an EMR system was associated with a sustainable reduction of LOS, while the DPC/PDPS was related to an increasing trend in LOS even though the level of LOS fell instantly post-DPC/PDPS implementation. We also suggest that the observed reduction of the ALOS in the long term might have resulted from the interaction of the EMR system with the DPC/PDPS. These findings suggest that EMRs could improve efficiency either by improving the practicability of other interventions and activities, such as hospital funding systems, or by contributing to improvements in the quality of healthcare, leading to reductions in LOS and cost. However, DPC/PDPS may not be effective at delivering its expected outcomes. Consequently, policymakers should consider both long- and short-term effects of these schemes instead of focusing on the shortterm results. It is also noteworthy that supporting medical care-related interventions with infrastructure that enables better implementation of interventions such as EMRs could lead to better healthcare outcomes.

For researchers, studies that evaluate the effectiveness of EMR and the DPC/PDPS on short- and long-term medical care outcomes need to be carried out based on data from multiple sites. A comparison of the effects of EMR and DPC/ PDPS on healthcare outcomes, such as LOS, for specific diseases/conditions or departments also could provide better information for evidence-based practice.

The primary strength of this study is that it applied the strongest quasi-experimental design, ITS analysis, which makes it possible to control for temporal confounders that could exist in the study period and provide more accurate estimates. We also made efforts to capture changes in LOS in the long term (11 years) and compared the short- and longterm effects of interventions.

The possible limitations of this study are as follows. First, the analysis was based on data from a single site and we could not focus on data from a specific disease or from specific departments, as our data would not be adequate to conduct an ITS analysis. Second, due to practical constraints, we could not use other hospitals that had not adopted EMRs and DPC/PDPS as controls. Instead, we used before/after 
control groups for the analysis. Third, although we made sure that no interventions that could significantly affect the outcome variable had taken place during the study period, it remains possible that unidentified non-temporal variables, such as changes in clinical guidelines, the severity of diseases, or socioeconomic status might have confounded the estimates in this study. These limitations should be considered when interpreting the results of this study.

\section{Conflict of Interest}

No potential conflict of interest relevant to this article was reported.

\section{Acknowledgments}

This work was supported by the JSPS KAKENHI (No. 19K21589). The funder had no role in the preparation, review, or approval of the manuscript or the decision to submit the manuscript for publication.

\section{ORCID}

Melaku Haile Likka (https://orcid.org/0000-0001-5650-728X) Yukio Kurihara (https://orcid.org/0000-0001-5145-4203)

\section{References}

1. Mihailovic N, Kocic S, Jakovljevic M. Review of diagnosis-related group-based financing of hospital care. Health Serv Res Manag Epidemiol 2016;3:2333392816647892.

2. Organization for Economic Co-operation and Development. Health at a glance 2019: OECD indicators. Paris, France: Organization for Economic Co-operation and Development; 2019.

3. Ishii M. DRG/PPS and DPC/PDPS as prospective payment systems. Japan Med Assoc J 2012;55(4):279-91.

4. Annear PL, Kwon S, Lorenzoni L, Duckett S, Huntington D, Langenbrunner JC, et al. Pathways to DRG-based hospital payment systems in Japan, Korea, and Thailand. Health Policy 2018;122(7):707-13.

5. Jang SI, Nam CM, Lee SG, Kim TH, Park S, Park EC. Impact of payment system change from per-case to perdiem on high severity patient's length of stay. Medicine (Baltimore) 2016;95(37):e4839.

6. O’Reilly J, Busse R, Hakkinen U, Or Z, Street A, Wiley M. Paying for hospital care: the experience with implementing activity-based funding in five European coun- tries. Health Econ Policy Law 2012;7(1):73-101.

7. Meng Z, Hui W, Cai Y, Liu J, Wu H. The effects of DRGs-based payment compared with cost-based payment on inpatient healthcare utilization: a systematic review and meta-analysis. Health Policy 2020;124(4):35967.

8. Jung YW, Pak H, Lee I, Kim EH. The effect of diagnosisrelated group payment system on quality of care in the field of obstetrics and gynecology among Korean tertiary hospitals. Yonsei Med J 2018;59(4):539-45.

9. Choi JW, Kim SJ, Park HK, Jang SI, Kim TH, Park EC. Effects of a mandatory DRG payment system in South Korea: analysis of multi-year nationwide hospital claims data. BMC Health Serv Res 2019;19(1):776.

10. Hamada H, Sekimoto M, Imanaka Y. Effects of the per diem prospective payment system with DRG-like grouping system (DPC/PDPS) on resource usage and healthcare quality in Japan. Health Policy 2012;107(23):194-201.

11. Kondo A, Kawabuchi K. Evaluation of the introduction of a diagnosis procedure combination system for patient outcome and hospitalisation charges for patients with hip fracture or lung cancer in Japan. Health Policy 2012; 107(2-3):184-93.

12. Jian W, Lu M, Chan KY, Poon AN, Han W, Hu M, et al. Payment reform pilot in Beijing hospitals reduced expenditures and out-of-pocket payments per admission. Health Aff (Millwood) 2015;34(10):1745-52.

13. Hu WY, Yeh CF, Shiao AS, Tu TY. Effects of diagnosisrelated group payment on health-care provider behaviors: a consecutive three-period study. J Chin Med Assoc 2015;78(11):678-85.

14. Kaneko K, Onozuka D, Shibuta H, Hagihara A. Impact of electronic medical records (EMRs) on hospital productivity in Japan. Int J Med Inform 2018;118:36-43.

15. Centers for Medicaid and Medicare Services. Electronic Health Records [Internet]. Baltimore (MD): Centers for Medicaid and Medicare Services; 2012 [cited at 2021 Oct 18]. Available from: https://www.cms.gov/Medicare/E-Health/EHealthRecords.

16. Japan Association of Medical Informatics. JAMI viewpoint concerning the definition of the electronic medical record [Internet]. Tokyo, Japan: Japan Association of Medical Informatics; 2003 [cited at 2021 Oct 18]. Available from: http://jami.jp/english/about/doc/denshikarute_en.pdf.

17. Thompson G, O'Horo JC, Pickering BW, Herasevich V. Impact of the electronic medical record on mortality, 
length of stay, and cost in the hospital and ICU: a systematic review and metaanalysis. Crit Care Med 2015; 43(6):1276-82.

18. Agha L. The effects of health information technology on the costs and quality of medical care. J Health Econ 2014;34:19-30.

19. Barnett ML, Mehrotra A, Jena AB. Adverse inpatient outcomes during the transition to a new electronic health record system: observational study. BMJ 2016; 354:i3835.

20. Yanamadala S, Morrison D, Curtin C, McDonald K, Hernandez-Boussard T. Electronic health records and quality of care: an observational study modeling impact on mortality, readmissions, and complications. Medicine (Baltimore) 2016;95(19):e3332.

21. Wani D, Malhotra M. Does the meaningful use of electronic health records improve patient outcomes? J Oper Manag 2018;60:1-18.

22. Yang P, Cao Y, Liu D, Bai Y, Pan F, Xu Y. The effect of electronic medical record application on the length of stay in a Chinese general hospital: a department- and disease-focused interrupted time-series study. J Med Syst 2014;38(5):53.

23. Xue Y, Liang H, Wu X, Gong H, Li B, Zhang Y. Effects of electronic medical record in a Chinese hospital: a time series study. Int J Med Inform 2012;81(10):683-9.

24. Freedman S, Lin H, Prince J. Information technology and patient health: analyzing outcomes, populations, and mechanisms. Am J Health Econ 2018;4(1):51-79.

25. Inokuchi R, Sato H, Nakamura K, Aoki Y, Shinohara K, Gunshin $\mathrm{M}$, et al. Motivations and barriers to implementing electronic health records and ED information systems in Japan. Am J Emerg Med 2014;32(7):725-30.

26. Kanakubo T, Kharrazi H. Comparing the trends of electronic health record adoption among hospitals of the United States and Japan. J Med Syst 2019;43(7):224.

27. Kawaguchi H, Koike S, Ohe K. Regional differences in electronic medical record adoption in Japan: a nationwide longitudinal ecological study. Int J Med Inform 2018;115:114-9.

28. Bernal JL, Cummins S, Gasparrini A. Interrupted time series regression for the evaluation of public health interventions: a tutorial. Int J Epidemiol 2017;46(1):34855.

29. Hategeka C, Ruton H, Karamouzian M, Lynd LD, Law MR. Use of interrupted time series methods in the evaluation of health system quality improvement interventions: a methodological systematic review. BMJ Glob Health 2020;5(10):e003567. 